\title{
L(2,1)-Labeling in the Context of Some Graph Operations
}

\author{
S K Vaidya (Corresponding author) \\ Department of Mathematics, Saurashtra University \\ Rajkot 360 005, Gujarat, India \\ E-mail: samirkvaidya@yahoo.co.in \\ P L Vihol \\ Mathematics Department, Government Polytechnic \\ Rajkot 360 003, Gujarat, India \\ E-mail: viholprakash@yahoo.com \\ N A Dani \\ Mathematics Department, Government Polytechnic \\ Junagadh 362 001, Gujarat, India \\ E-mail: nilesh_a_d@yahoo.co.in
}

D D Bantva

Mathematics Department, Atmiya Institute of Technology and Science

Rajkot 360 005, Gujarat, India

E-mail: devsi.bantva@gmail.com

\begin{abstract}
Let $G=(V(G), E(G))$ be a connected graph. For integers $j \geq k, L(j, k)$-labeling of a graph $G$ is an integer labeling of the vertices in $\mathrm{V}$ such that adjacent vertices receive integers which differ by at least $j$ and vertices which are at distance two apart receive labels which differ by at least $k$. In this paper we discuss $L(2,1)$-labeling (or distance two labeling) in the context of some graph operations.
\end{abstract}

Keywords: Graph Labeling, $\lambda$ - Number, $\lambda^{\prime}$ - Number

\section{Introduction}

We begin with finite, connected, undirected graph $G=(V(G), E(G))$ without loops and multiple edges. For standard terminology and notations we refer to (West, D., 2001). We will give brief summary of definitions and information which are prerequisites for the present work.

Definition 1.1 Duplication of a vertex $v_{k}$ of graph $G$ produces a new graph $G^{\prime}$ by adding a vertex $v_{k}^{\prime}$ with $N\left(v_{k}^{\prime}\right)=N\left(v_{k}\right)$. In other words a vertex $v_{k}^{\prime}$ is said to be duplication of $v_{k}$ if all the vertices which are adjacent to $v_{k}$ are now adjacent to $v_{k}^{\prime}$ also.

Definition 1.2 Let $G$ be a graph. A graph $H$ is called a supersubdivision of $G$ if $H$ is obtained from $G$ by replacing every edge $e_{i}$ of $G$ by a complete bipartite graph $K_{2, m_{i}}$ (for some $m_{i}$ and $1 \leq i \leq q$ ) in such a way that the ends of each $e_{i}$ are merged with the two vertices of 2-vertices part of $K_{2, m_{i}}$ after removing the edge $e_{i}$ from graph $G$.

A new family of graph introduced in (Vaidya, S., 2008, p.54-64) defined as follows.

Definition 1.3 A graph obtained by replacing each vertex of a star $K_{1, n}$ by a graph $G$ is called star of $G$ denoted as $G^{\prime}$. The central graph in $G^{\prime}$ is the graph which replaces apex vertex of $K_{1, n}$.

Definition 1.4 If the vertices of the graph are assigned values subject to certain conditions then it is known as graph labeling.

The unprecedented growth of wireless communication is recorded but the available radio frequencies allocated to these communication networks are not enough. Proper allocation of frequencies is demand of the time. The interference by unconstrained transmitters will interrupt the communications. This problem was taken up in (Hale, W., 1980, p.14971514) in terms of graph labeling. In a private communication with Griggs during 1988 Roberts proposed a variation in channel assignment problem. According to him any two close transmitters must receive different channels in order to avoid interference. Motivated by this problem the concept of L(2,1)-labeling was introduced by (Yeh, R., 1990) and 
(Griggs, J., and Yeh, R., 1992, p.586-595) which is defined as follows.

Definition 1.5 For a graph $G, L(2,1)$-labeling (or distance two labeling) with span $k$ is a function $f: V(G) \longrightarrow\{0,1, \ldots, k\}$ such that the following conditions are satisfied:

(1) $|f(x)-f(y)| \geq 2$ if $d(x, y)=1$

(2) $|f(x)-f(y)| \geq 1$ if $d(x, y)=2$

In otherwords the $L(2,1)$-labeling of a graph is an abstraction of assigning integer frequencies to radio transmitters such that (1) Transmitters that are one unit of distance apart receive frequencies that differ by at least two and (2) Transmitters that are two units of distance apart receive frequencies that differ by at least one. The span of $f$ is the largest number in $f(V)$. The minimum span taken over all $L(2,1)$-labeling of $G$, denoted as $\lambda(G)$ is called the $\lambda$-number of $G$. The minimum label in $L(2,1)$-labeling of $G$ is assumed to be 0 .

Definition 1.6 An injective $L(2,1)$-labeling is called an $L^{\prime}(2,1)$-labeling and the minimum span taken over all such $L^{\prime}(2,1)$-labeling is called $\lambda^{\prime}$-number of the graph.

The L(2,1)-labeling has been extensively studied in the recent past by many researchers (Georges, J., 1995, p.141-159), (Georges, J.P., 2001, p.28-35),(Georges, J., 1996, p.47-57),(Georges, J., 1994, p.103-111),(Liu. D., 1997, p.13-22),(Shao. Z., 2005, p.668-671). Practically it is observed that the interference might go beyond two levels. This observation motivated (Chartrand. G., 2001, p.77-85) to introduce the concept of radio labeling which is the extension of $L(2,1)$ labeling when the interference is beyond two levels to the largest possible - the diameter of $G$. We investigate three results corresponding to $L(2,1)$-labeling and $L^{\prime}(2,1)$-labeling each.

\section{Main Results}

Theorem 2.1 Let $C_{n}^{\prime}$ be the graph obtained by duplicating all the vertices of the cycle $C_{n}$ at a time then $\lambda\left(C_{n}^{\prime}\right)=7$. (where $n>3)$

Proof: Let $v_{1}^{\prime}, v_{2}^{\prime}, \ldots, v_{n}^{\prime}$ be the duplicated vertices corresponding to $v_{1}, v_{2}, \ldots, v_{n}$ of cycle $C_{n}$.

To define $f: V\left(C_{n}^{\prime}\right) \longrightarrow N \bigcup\{0\}$, we consider following four cases.

Case 1: $n \equiv 0(\bmod 3)($ where $n>5)$

We label the vertices as follows.

$$
\begin{gathered}
f\left(v_{i}\right)=0, i=3 j-2,1 \leq j \leq \frac{n}{3} \\
f\left(v_{i}\right)=2, i=3 j-1,1 \leq j \leq \frac{n}{3} \\
f\left(v_{i}\right)=4, i=3 j, 1 \leq j \leq \frac{n}{3} \\
f\left(v_{i}^{\prime}\right)=7, i=3 j-2,1 \leq j \leq \frac{n}{3} \\
f\left(v_{i}^{\prime}\right)=6, i=3 j-1,1 \leq j \leq \frac{n}{3} \\
f\left(v_{i}^{\prime}\right)=5, i=3 j, 1 \leq j \leq \frac{n}{3}
\end{gathered}
$$

Case 2: $n \equiv 1(\bmod 3)($ where $n>5)$

We label the vertices as follows.

$$
\begin{gathered}
f\left(v_{i}\right)=0, i=3 j-2,1 \leq j \leq\left\lfloor\frac{n}{3}\right\rfloor \\
f\left(v_{i}\right)=2, i=3 j-1,1 \leq j \leq\left\lfloor\frac{n}{3}\right\rfloor \\
f\left(v_{i}\right)=4, i=3 j, 1 \leq j \leq\left\lfloor\frac{n}{3}\right\rfloor \\
f\left(v_{n-3}\right)=0, f\left(v_{n-2}\right)=3, f\left(v_{n-1}\right)=1, f\left(v_{n}\right)=4 \\
f\left(v_{i}^{\prime}\right)=7, i=3 j-2,1 \leq j \leq\left\lfloor\frac{n}{3}\right\rfloor \\
f\left(v_{i}^{\prime}\right)=6, i=3 j-1,1 \leq j \leq\left\lfloor\frac{n}{3}\right\rfloor \\
f\left(v_{i}^{\prime}\right)=5, i=3 j, 1 \leq j \leq\left\lfloor\frac{n}{3}\right\rfloor \\
f\left(v_{n-3}^{\prime}\right)=7, f\left(v_{n-2}^{\prime}\right)=7, f\left(v_{n-1}^{\prime}\right)=6, f\left(v_{n}^{\prime}\right)=5
\end{gathered}
$$

Case 3: $n \equiv 2(\bmod 3)($ where $n>5)$

We label the vertices as follows.

$$
\begin{gathered}
f\left(v_{i}\right)=0, i=3 j-2,1 \leq j \leq\left\lfloor\frac{n}{3}\right\rfloor \\
f\left(v_{i}\right)=2, i=3 j-1,1 \leq j \leq\left\lfloor\frac{n}{3}\right\rfloor \\
f\left(v_{i}\right)=4, i=3 j, 1 \leq j \leq\left\lfloor\frac{n}{3}\right\rfloor \\
f\left(v_{n-1}\right)=1, f\left(v_{n}\right)=3 \\
f\left(v_{1}^{\prime}\right)=6, f\left(v_{n}^{\prime}\right)=7
\end{gathered}
$$




$$
\begin{gathered}
f\left(v_{i}^{\prime}\right)=6, i=3 j-1,1 \leq j \leq\left\lfloor\frac{n}{3}\right\rfloor \\
f\left(v_{i}^{\prime}\right)=5, i=3 j, 1 \leq j \leq\left\lfloor\frac{n}{3}\right\rfloor \\
f\left(v_{i}^{\prime}\right)=7, i=3 j+1,1 \leq j \leq\left\lfloor\frac{n}{3}\right\rfloor
\end{gathered}
$$

Case 4: $n=4,5$

These cases are to be dealt separately. The $L(2,1)$-labeling for the graphs obtained by duplicating all the vertices at a time in the cycle $C_{n}$ when $n=4,5$ are as shown in Fig 1

Thus in all the possibilities $R_{f}=\{0,1,2 \ldots, 7\} \subset N \cup\{0\}$.

i.e. $\lambda\left(C_{n}^{\prime}\right)=7$.

Remark The $L(2,1)$-labeling for the graph obtained by duplicating all the vertices of the cycle $C_{3}$ is shown in Fig 2 Thus $R_{f}=\{0,1,2 \ldots, 6\} \subset N \bigcup\{0\}$.

i.e. $\lambda\left(C_{3}^{\prime}\right)=6$.

Illustration 2.2 Consider the graph $C_{6}$ and duplicate all the vertices at a time. The L(2,1)-labeling is as shown in Fig 3 .

Theorem 2.3 Let $C_{n}^{\prime}$ be the graph obtained by duplicating all the vertices at a time of the cycle $C_{n}$ then $\lambda^{\prime}\left(C_{n}^{\prime}\right)=p-1$, where $p$ is the total number vertices in $C_{n}^{\prime}$ (where $n>3$ ).

Proof: Let $v_{1}^{\prime}, v_{2}^{\prime}, \ldots, v_{n}^{\prime}$ be the duplicated vertices corresponding to $v_{1}, v_{2}, \ldots, v_{n}$ of cycle $C_{n}$.

To define $f: V\left(C_{n}^{\prime}\right) \longrightarrow N \cup\{0\}$, we consider following two cases.

Case 1: $n>5$

$$
\begin{gathered}
f\left(v_{i}\right)=2 i-7,4 \leq i \leq n \\
f\left(v_{i}\right)=f\left(v_{n}\right)+2,1 \leq i \leq 3 \\
f\left(v_{i}^{\prime}\right)=2 i-2,1 \leq i \leq n
\end{gathered}
$$

Now label the vertices of $C_{n}^{\prime}$ using the above defined pattern we have $R_{f}=\{0,1,2, \ldots, p-1\} \subset N \bigcup\{0\}$

This implies that $\lambda^{\prime}\left(C_{n}^{\prime}\right)=p-1$.

Case 2: $n=4,5$

These cases to be dealt separately. The $L^{\prime}(2,1)$-labeling for the graphs obtained by duplicating all the vertices at a time in the cycle $C_{n}$ when $n=4,5$ are as shown in the following Fig 4 .

Remark The $L^{\prime}(2,1)$-labeling for the graphs obtained by duplicating all the vertices at a time in the cycle $C_{3}$ is shown in the following Fig 5 .

Thus $R_{f}=\{0,1,2 \ldots, 6\} \subset N \bigcup\{0\}$.

i.e. $\lambda^{\prime}\left(C_{3}^{\prime}\right)=6$.

Illustration 2.4 Consider the graph $C_{6}$ and duplicating all the vertices at a time. The $L^{\prime}(2,1)$-labeling is as shown in Fig 6.

Theorem 2.5 Let $C_{n}^{\prime}$ be the graph obtained by taking arbitrary supersubdivision of each edge of cycle $C_{n}$ then

1 For $n$ even

$$
\lambda\left(C_{n}^{\prime}\right)=\Delta+2
$$

2 For $n$ odd

$$
\lambda\left(C_{n}^{\prime}\right)=\left\{\begin{array}{lr}
\Delta+2 ; & \text { if } s+t+r<\Delta, \\
\Delta+3 ; & \text { if } s+t+r=\Delta, \\
s+t+r+2 ; & \text { if } s+t+r>\Delta
\end{array}\right.
$$

where $v_{k}$ is a vertex with label 2 ,

$s$ is number of subdivision between $v_{k-2}$ and $v_{k-1}$,

$t$ is number of subdivision between $v_{k-1}$ and $v_{k}$,

$r$ is number of subdivision between $v_{k}$ and $v_{k+1}$,

$\Delta$ is the maximum degree of $C_{n}^{\prime}$.

Proof: Let $v_{1}, v_{2}, \ldots, v_{n}$ be the vertices of cycle $C_{n}$. Let $C_{n}^{\prime}$ be the graph obtained by arbitrary super subdivision of cycle $C_{n}$. 
It is obvious that for any two vertices $v_{i}$ and $v_{i+2}, N\left(v_{i}\right) \bigcap N\left(v_{i+2}\right)=\phi$

To define $f: V\left(C_{n}^{\prime}\right) \longrightarrow N \bigcup\{0\}$, we consider following two cases.

Case 1: $C_{n}$ is even cycle

$$
\begin{gathered}
f\left(v_{2 i-1}\right)=0,1 \leq i \leq \frac{n}{2} \\
f\left(v_{2 i}\right)=1,1 \leq i \leq \frac{n}{2}
\end{gathered}
$$

If $P_{i j}$ is the number of supersubdivisions between $v_{i}$ and $v_{j}$ then for the vertex $v_{1},\left|N\left(v_{1}\right)\right|=P_{12}+P_{n 1}$. Without loss of generality we assume that $v_{1}$ is the vertex with maximum degree i.e. $d\left(v_{1}\right)=\Delta$. suppose $u_{1}, u_{2} \ldots . . u_{\Delta}$ be the members of $N\left(v_{1}\right)$. We label the vertices of $N\left(v_{1}\right)$ as follows.

$f\left(u_{i}\right)=2+i, 1 \leq i \leq \Delta$

As $N\left(v_{1}\right) \bigcap N\left(v_{3}\right)=\phi$ then it is possible to label the vertices of $N\left(v_{3}\right)$ using the vertex labels of the members of $N\left(v_{1}\right)$ in accordance with the requirement for $L(2,1)$-labeling. Extending this argument recursively upto $N\left(v_{n-1}\right)$ it is possible to label all the vertices of $C_{n}^{\prime}$ using the distinct numbers between 0 and $\Delta+2$.

i.e. $R_{f}=\{0,1,2, \ldots, \Delta+2\} \subset N \bigcup\{0\}$

Consequently $\lambda\left(C_{n}^{\prime}\right)=\Delta+2$.

Case 2: $C_{n}$ is odd cycle

Let $v_{1}, v_{2}, \ldots, v_{n}$ be the vertices of cycle $C_{n}$.

Without loss of generality we assume that $v_{1}$ is a vertex with maximum degree and $v_{k}$ be the vertex with minimum degree.

Define $f\left(v_{k}\right)=2$ and label the remaining vertices alternatively with labels 0 and 1 such that $f\left(v_{1}\right)=0$. Then either $f\left(v_{k-1}\right)=1 ; f\left(v_{k+1}\right)=0$ OR $f\left(v_{k-1}\right)=0 ; f\left(v_{k+1}\right)=1$. We assign labeling in such a way that $f\left(v_{k-1}\right)=1 ; f\left(v_{k+1}\right)=0$.

Now following the procedure adapted in case (1) it is possible to label all the vertices except the vertices between $v_{k-1}$ and $v_{k}$. Label the vertices between $v_{k-1}$ and $v_{k}$ using the vertex labels of $N\left(v_{1}\right)$ except the labels which are used earlier to label the vertices between $v_{k-2}, v_{k-1}$ and between $v_{k}, v_{k+1}$.

If there are $p$ vertices $u_{1}, u_{2} \ldots u_{p}$ are left unlabeled between $v_{k-1}$ and $v_{k}$ then label them as follows,

$f\left(u_{i}\right)=\max \left\{\right.$ labels of the vertices between $v_{k-2}$ and $v_{k-1}$, labels of the vertices between $v_{k}$ and $\left.v_{k+1}\right\}+i, 1 \leq i \leq p$

Now if $s$ is the number of subdivisions between $v_{k-2}$ and $v_{k-1}$

$t$ is the number of subdivisions between $v_{k-1}$ and $v_{k}$

$r$ is the number of subdivisions between $v_{k}$ and $v_{k+1}$

then (1) $R_{f}=\{0,1,2, \ldots, \Delta+2\} \subset N \bigcup\{0\}$, when $s+t+r<\Delta$

i.e. $\lambda\left(C_{n}^{\prime}\right)=\Delta+2$

(2) $R_{f}=\{0,1,2, \ldots, \Delta+3\} \subset N \bigcup\{0\}$, when $s+t+r=\Delta$

i.e. $\lambda\left(C_{n}^{\prime}\right)=\Delta+3$

(3) $R_{f}=\{0,1,2, \ldots, s+t+r+2\} \subset N \bigcup\{0\}$, when $s+t+r>\Delta$

i.e. $\lambda\left(C_{n}^{\prime}\right)=s+t+r+2$

Illustration 2.6 Consider the graph $C_{8}$. The $L(2,1)$-labeling of $C_{8}^{\prime}$ is shown in Fig 7 .

Theorem 2.7 Let $G^{\prime}$ be the graph obtained by taking arbitrary supersubdivision of each edge of graph $G$ with number of vertices $n \geq 3$ then $\lambda^{\prime}\left(G^{\prime}\right)=p-1$, where $p$ is the total number of vertices in $G^{\prime}$.

Proof: Let $v_{1}, v_{2}, \ldots, v_{n}$ be the vertices of any connected graph $G$ and let $G^{\prime}$ be the graph obtained by taking arbitrary supersubdivision of $G$. Let $u_{k}$ be the vertices which are used for arbitrary supersubdivision of the edge $v_{i} v_{j}$ where $1 \leq i \leq$ $n, 1 \leq j \leq n$ and $i<j$

Here $k$ is a total number of vertices used for arbitrary supersubdivision.

We define $f: V\left(G^{\prime}\right) \longrightarrow N \cup\{0\}$ as

$f\left(v_{i}\right)=i-1$, where $1 \leq i \leq n$

Now we label the vertices $u_{i}$ in the following order.

First we label the vertices between $v_{1}$ and $v_{1+j}, 1 \leq j \leq n$ then following the same procedure for $v_{2}, v_{3}, \ldots v_{n}$

$f\left(u_{i}\right)=f\left(v_{n}\right)+i, 1 \leq i \leq k$

Now label the vertices of $G^{\prime}$ using the above defined pattern we have $R_{f}=\{0,1,2, \ldots, p-1\} \subset N \bigcup\{0\}$ 
This implies that $\lambda^{\prime}\left(G^{\prime}\right)=p-1$.

Illustration 2.8 Consider the graph $P_{4}$ and its supersubdivision. The $L^{\prime}(2,1)$-labeling is as shown in Fig 8 .

Theorem 2.9 Let $C_{n}^{\prime}$ be the graph obtained by taking star of a cycle $C_{n}$ then $\lambda\left(C_{n}^{\prime}\right)=5$.

Proof:Let $v_{1}, v_{2}, \ldots, v_{n}$ be the vertices of cycle $C_{n}$ and $v_{i j}$ be the vertices of cycle $C_{n}$ which are adjacent to the $i^{t h}$ vertex of cycle $C_{n}$.

To define $f: V\left(C_{n}^{\prime}\right) \longrightarrow N \bigcup\{0\}$, we consider following four cases.

Case 1: $n \equiv 0(\bmod 3)$

$$
\begin{gathered}
f\left(v_{i}\right)=0, i=3 j-2,1 \leq j \leq \frac{n}{3} \\
f\left(v_{i}\right)=2, i=3 j-1,1 \leq j \leq \frac{n}{3} \\
f\left(v_{i}\right)=4, i=3 j, 1 \leq j \leq \frac{n}{3}
\end{gathered}
$$

Now we label the vertices $v_{i j}$ of star of a cycle according to the label of $f\left(v_{i}\right)$.

(1) when $f\left(v_{i}\right)=0, i=3 j-2,1 \leq j \leq \frac{n}{3}$

$$
\begin{gathered}
f\left(v_{i k}\right)=3, k=3 p-2,1 \leq p \leq \frac{n}{3} \\
f\left(v_{i k}\right)=5, k=3 p-1,1 \leq p \leq \frac{n}{3} \\
f\left(v_{i k}\right)=1, k=3 p, 1 \leq p \leq \frac{n}{3}
\end{gathered}
$$

(2) when $f\left(v_{i}\right)=2, i=3 j-1,1 \leq j \leq \frac{n}{3}$

$$
\begin{gathered}
f\left(v_{i k}\right)=5, k=3 p-2,1 \leq p \leq \frac{n}{3} \\
f\left(v_{i k}\right)=3, k=3 p-1,1 \leq p \leq \frac{n}{3} \\
f\left(v_{i k}\right)=1, k=3 p, 1 \leq p \leq \frac{n}{3}
\end{gathered}
$$

(3) when $f\left(v_{i}\right)=4, i=3 j, 1 \leq j \leq \frac{n}{3}$

$$
\begin{gathered}
f\left(v_{i k}\right)=1, k=3 p-2,1 \leq p \leq \frac{n}{3} \\
f\left(v_{i k}\right)=3, k=3 p-1,1 \leq p \leq \frac{n}{3} \\
f\left(v_{i k}\right)=5, k=3 p, 1 \leq p \leq \frac{n}{3}
\end{gathered}
$$

Case 2: $n \equiv 1(\bmod 3)$

$$
\begin{gathered}
f\left(v_{i}\right)=0, i=3 j-2,1 \leq j \leq\left\lfloor\frac{n}{3}\right\rfloor \\
f\left(v_{i}\right)=2, i=3 j-1,1 \leq j \leq\left\lfloor\frac{n}{3}\right\rfloor \\
f\left(v_{i}\right)=5, i=3 j, 1 \leq j \leq\left\lfloor\frac{n}{3}\right\rfloor \\
f\left(v_{n}\right)=3
\end{gathered}
$$

Now we label the vertices of star of a cycle $v_{i j}$ according to label of $f\left(v_{i}\right)$.

(1) when $f\left(v_{i}\right)=0, i=3 j-2,1 \leq j \leq\left\lfloor\frac{n}{3}\right\rfloor$

$$
\begin{gathered}
f\left(v_{i k}\right)=4, k=3 p-2,1 \leq p \leq\left\lfloor\frac{n}{3}\right\rfloor \\
f\left(v_{i k}\right)=2, k=3 p-1,1 \leq p \leq\left\lfloor\frac{n}{3}\right\rfloor \\
f\left(v_{i k}\right)=0, k=3 p, 1 \leq p \leq\left\lfloor\frac{n}{3}\right\rfloor-1 \\
f\left(v_{i(n-1)}\right)=5 \\
f\left(v_{i n}\right)=1
\end{gathered}
$$

(2) when $f\left(v_{i}\right)=2, i=3 j-1,1 \leq j \leq\left\lfloor\frac{n}{3}\right\rfloor$

$$
\begin{gathered}
f\left(v_{i k}\right)=4, k=3 p-2,1 \leq p \leq\left\lfloor\frac{n}{3}\right\rfloor \\
f\left(v_{i k}\right)=0, k=3 p-1,1 \leq p \leq\left\lfloor\frac{n}{3}\right\rfloor \\
f\left(v_{i k}\right)=2, k=3 p, 1 \leq p \leq\left\lfloor\frac{n}{3}\right\rfloor-1 \\
f\left(v_{i(n-1)}\right)=3, \\
f\left(v_{i n}\right)=1
\end{gathered}
$$

(3) when $f\left(v_{i}\right)=5, i=3 j, 1 \leq j \leq\left\lfloor\frac{n}{3}\right\rfloor$ 


$$
\begin{gathered}
f\left(v_{i k}\right)=1, k=3 p-2,1 \leq p \leq\left\lfloor\frac{n}{3}\right\rfloor \\
f\left(v_{i k}\right)=3, k=3 p-1,1 \leq p \leq\left\lfloor\frac{n}{3}\right\rfloor \\
f\left(v_{i k}\right)=5, k=3 p, 1 \leq p \leq\left\lfloor\frac{n}{3}\right\rfloor-1 \\
f\left(v_{i(n-1)}\right)=0, \\
f\left(v_{i n}\right)=4
\end{gathered}
$$

(4) when $f\left(v_{i}\right)=3, i=n$

$$
\begin{gathered}
f\left(v_{i k}\right)=1, k=3 p-2,1 \leq p \leq\left\lfloor\frac{n}{3}\right\rfloor \\
f\left(v_{i k}\right)=5, k=3 p-1,1 \leq p \leq\left\lfloor\frac{n}{3}\right\rfloor \\
f\left(v_{i k}\right)=3, k=3 p, 1 \leq p \leq\left\lfloor\frac{n}{3}\right\rfloor-1 \\
f\left(v_{i(n-1)}\right)=0 \\
f\left(v_{i n}\right)=4
\end{gathered}
$$

Case 3: $n \equiv 2(\bmod 3), n \neq 5$

$$
\begin{gathered}
f\left(v_{i}\right)=1, i=3 j-2,1 \leq j \leq\left\lfloor\frac{n}{3}\right\rfloor-1 \\
f\left(v_{i}\right)=3, i=3 j-1,1 \leq j \leq\left\lfloor\frac{n}{3}\right\rfloor-1 \\
f\left(v_{i}\right)=5, i=3 j, 1 \leq j \leq\left\lfloor\frac{n}{3}\right\rfloor-1 \\
f\left(v_{n-4}\right)=0, f\left(v_{n-3}\right)=2, f\left(v_{n-2}\right)=5, f\left(v_{n-1}\right)=0, f\left(v_{n}\right)=4
\end{gathered}
$$

Now we label the vertices $v_{i j}$ of star of a cycle according to the label of $f\left(v_{i}\right)$.

(1) when $f\left(v_{i}\right)=1, i=3 j-2,1 \leq j \leq\left\lfloor\frac{n}{3}\right\rfloor-1$

$$
\begin{gathered}
f\left(v_{i k}\right)=5, k=3 p-2,1 \leq p \leq\left\lfloor\frac{n}{3}\right\rfloor \\
f\left(v_{i k}\right)=3, k=3 p-1,1 \leq p \leq\left\lfloor\frac{n}{3}\right\rfloor \\
f\left(v_{i k}\right)=1, k=3 p, 1 \leq p \leq\left\lfloor\frac{n}{3}\right\rfloor \\
f\left(v_{i(n-1)}\right)=4, \\
f\left(v_{i n}\right)=0
\end{gathered}
$$

(2) when $f\left(v_{i}\right)=3, i=3 j-1,1 \leq j \leq\left\lfloor\frac{n}{3}\right\rfloor-1$

$$
\begin{gathered}
f\left(v_{i k}\right)=0, k=3 p-2,1 \leq p \leq\left\lfloor\frac{n}{3}\right\rfloor \\
f\left(v_{i k}\right)=2, k=3 p-1,1 \leq p \leq\left\lfloor\frac{n}{3}\right\rfloor \\
f\left(v_{i k}\right)=4, k=3 p, 1 \leq p \leq\left\lfloor\frac{n}{3}\right\rfloor \\
f\left(v_{i(n-1)}\right)=1, \\
f\left(v_{i n}\right)=5
\end{gathered}
$$

(3) when $f\left(v_{i}\right)=5, i=3 j, 1 \leq j \leq\left\lfloor\frac{n}{3}\right\rfloor-1$ and $i=n-2$

$$
\begin{gathered}
f\left(v_{i k}\right)=1, k=3 p-2,1 \leq p \leq\left\lfloor\frac{n}{3}\right\rfloor \\
f\left(v_{i k}\right)=3, k=3 p-1,1 \leq p \leq\left\lfloor\frac{n}{3}\right\rfloor \\
f\left(v_{i k}\right)=5, k=3 p, 1 \leq p \leq\left\lfloor\frac{n}{3}\right\rfloor \\
f\left(v_{i(n-1)}\right)=2, \\
f\left(v_{i n}\right)=4
\end{gathered}
$$

(4) when $f\left(v_{i}\right)=0, i=n-4, n-1$

$$
\begin{gathered}
f\left(v_{i k}\right)=3, k=3 p-2,1 \leq p \leq\left\lfloor\frac{n}{3}\right\rfloor \\
f\left(v_{i k}\right)=5, k=3 p-1,1 \leq p \leq\left\lfloor\frac{n}{3}\right\rfloor \\
f\left(v_{i k}\right)=1, k=3 p, 1 \leq p \leq\left\lfloor\frac{n}{3}\right\rfloor-1 \\
f\left(v_{i(n-2)}\right)=2, \\
f\left(v_{i(n-1)}\right)=4, \\
f\left(v_{i n}\right)=1
\end{gathered}
$$

(5) when $f\left(v_{i}\right)=2, i=n-3$ 


$$
\begin{gathered}
f\left(v_{i k}\right)=4, k=3 p-2,1 \leq p \leq\left\lfloor\frac{n}{3}\right\rfloor \\
f\left(v_{i k}\right)=0, k=3 p-1,1 \leq p \leq\left\lfloor\frac{n}{3}\right\rfloor \\
f\left(v_{i k}\right)=2, k=3 p, 1 \leq p \leq\left\lfloor\frac{n}{3}\right\rfloor \\
f\left(v_{i(n-1)}\right)=5, \\
f\left(v_{i n}\right)=1
\end{gathered}
$$

(6) when $f\left(v_{i}\right)=4, i=n$

$$
\begin{gathered}
f\left(v_{i k}\right)=2, k=3 p-2,1 \leq p \leq\left\lfloor\frac{n}{3}\right\rfloor \\
f\left(v_{i k}\right)=0, k=3 p-1,1 \leq p \leq\left\lfloor\frac{n}{3}\right\rfloor \\
f\left(v_{i k}\right)=4, k=3 p, 1 \leq p \leq\left\lfloor\frac{n}{3}\right\rfloor \\
f\left(v_{i(n-1)}\right)=1, \\
f\left(v_{i n}\right)=5
\end{gathered}
$$

Case 4: $n=5$

This case is to be dealt separately. The $L(2,1)$-labeling for the graph obtained by taking star of the cycle $C_{5}$ is shown in Fig 9. Thus in all the possibilities we have $\lambda\left(C_{n}^{\prime}\right)=5$

Illustration 2.10 Consider the graph $C_{7}$, the $L(2,1)$-labeling is as shown in Fig 10 .

Theorem 2.11 Let $G^{\prime}$ be the graph obtained by taking star of a graph $G$ then $\lambda^{\prime}\left(G^{\prime}\right)=p-1$, where $p$ be the total number of vertices of $G^{\prime}$.

Proof: Let $v_{1}, v_{2}, \ldots, v_{n}$ be the vertices of any connected graph $G$. Let $v_{i j}$ be the vertices of a graph which is adjacent to the $i^{\text {th }}$ vertex of graph $G$. By the definition of a star of a graph the total number of vertices in a graph $G^{\prime}$ are $n(n+1)$.

To define $f: V\left(G^{\prime}\right) \longrightarrow N \bigcup\{0\}$

$f\left(v_{i 1}\right)=i-1, \quad 1 \leq i \leq n$

for $1 \leq i \leq n$ do the labeling as follows:

$$
\begin{gathered}
f\left(v_{i}\right)=f\left(v_{n i}\right)+1 \\
f\left(v_{1(i+1)}\right)=f\left(v_{i}\right)+1 \\
f\left(v_{(j+1)(i+1)}\right)=f\left(v_{j(i+1)}\right)+1,1 \leq j \leq n-1
\end{gathered}
$$

Thus $\lambda^{\prime}\left(G^{\prime}\right)=p-1=n^{2}+n-1$

Illustration 2.12 Consider the star of a graph $K_{4}$, the $L^{\prime}(2,1)$-labeling is shown in Fig 11 .

\section{Concluding Remarks}

Here we investigate some new results corresponding to $L(2,1)$-labeling and $L^{\prime}(2,1)$-labeling. The $\lambda$-number is completely determined for the graphs obtained by duplicating the vertices altogether in a cycle, arbitrary supersubdivision of a cycle and star of a cycle. We also determine $\lambda^{\prime}$-number for some graph families. This work is an effort to relate some graph operations and $L(2,1)$-labeling. All the results reported here are of very general nature and $\lambda$-number as well as $\lambda^{\prime}$-number are completely determined for the larger graphs resulted from the graph operations on standard graphs which is the salient features of this work. It is also possible to investigate some more results corresponding to other graph families.

\section{Acknowledgement}

The authors are thankful to the anonymous referee for useful suggestions and comments.

\section{References}

Chartrand, G. , Erwin, D. , Harary, F. \& Zhang P. (2001). Radio labeling of graphs. Bull, Inst. Combin. Appl, 33, 77-85.

Georges, J. \& Mauro, D. (1995). Generalized vertex labelings with a condition at distance two. Congr. Numer,109, 141-159.

Georges, J. P. , Mauro, D.W. \& Stein,M.I. (2001). Labeling products of complete graphs with a condition at distance two. SIAM J.Discrete Math, 14, 28-35.

Georges, J. , Mauro, D. \& Whittlesey, M. (1996). On the size of graphs labeled with a condition at distance two. J.Graph Theory, 22, 47-57.

Georges, J., Mauro, D. \& Whittlesey, M. (1994). Relating path covering to vertex labeling with a condition of distance two. Discrete Math, 135, 103-111.

Griggs, J.R. \& Yeh, R.K. (1992). Labeling graphs with a condition at distance two. SIAM J.Disc.Math, 5, 586-595. 
Hale, W.K. (1980). Frequency assignment: Theory and application. Proc.IEEE, 68, 1497-1514.

Liu, D. \& Yeh, R.K. (1997). On distance two labelings of graphs. Ars Combin, 47, 13-22.

Shao, Z. \& Yeh, R.K. (2005). The L(2,1)-labeling and operations of Graphs. IEEE Transactions on Circuits and Systems, 52(3), 668-671.

Vaidya, S.K., Ghodasara, G.V., Srivastav, S. \& Kaneria, V.J. (2008). Cordial and 3-equitable labeling of star of a cycle. Mathematics Today, 24, 54-64.

West, D.B. (2001). Introduction To Graph Theory, Prentice-Hall of India.

Yeh, R.K. (1990). Labeling Graphs with a Condition at Distance Two. Ph.D.dissertation, Dept.Math.,Univ.of South Carolina,Columbia, SC.
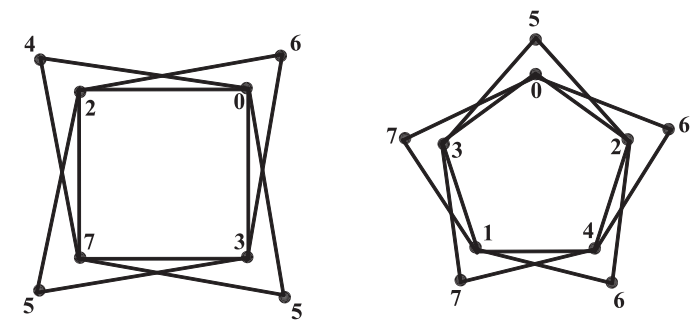

Figure 1. vertex duplication in $C_{4}, C_{5}$ and $L(2,1)$-labeling

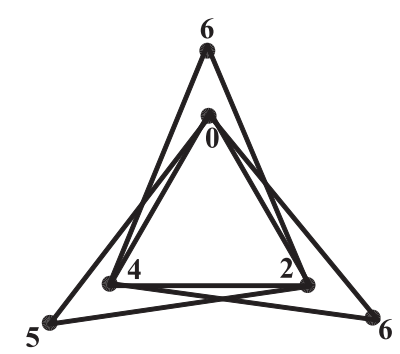

Figure 2. vertex duplication in $C_{3}$ and $L(2,1)$-labeling

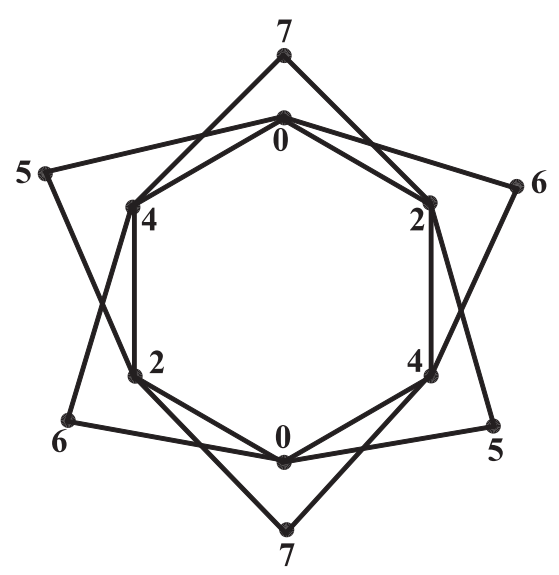

Figure 3. vertex duplication in $C_{6}$ and $L(2,1)$-labeling 

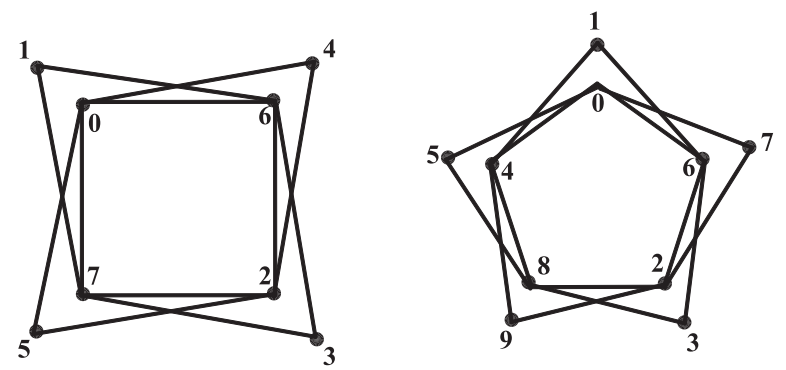

Figure 4. vertex duplication in $C_{4}, C_{5}$ and $L^{\prime}(2,1)$-labeling

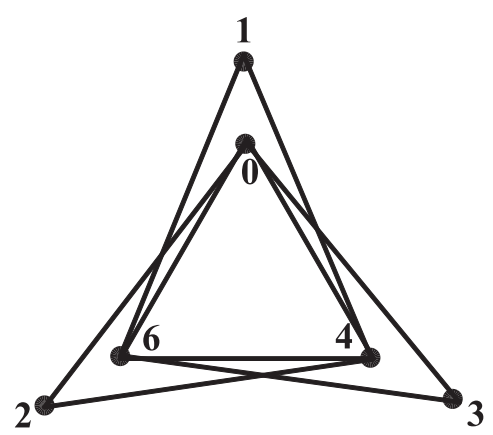

Figure 5. vertex duplication in $C_{3}$ and $L^{\prime}(2,1)$-labeling

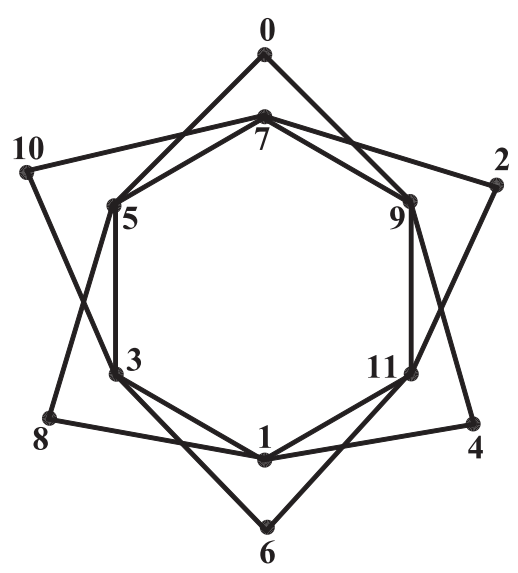

Figure 6. vertex duplication in $C_{6}$ and $L^{\prime}(2,1)$-labeling 


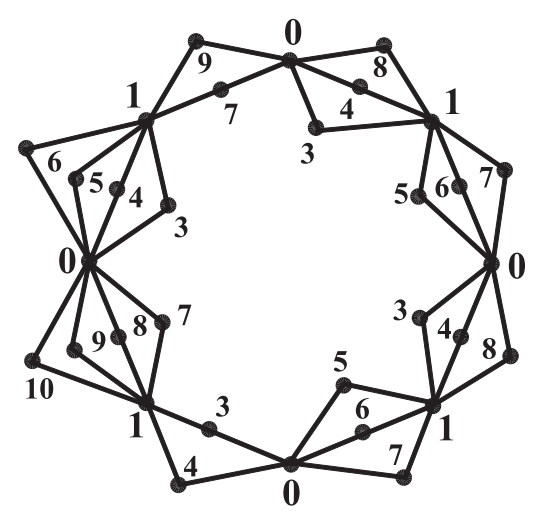

Figure 7. $L(2,1)$-labeling of $C_{8}^{\prime}$

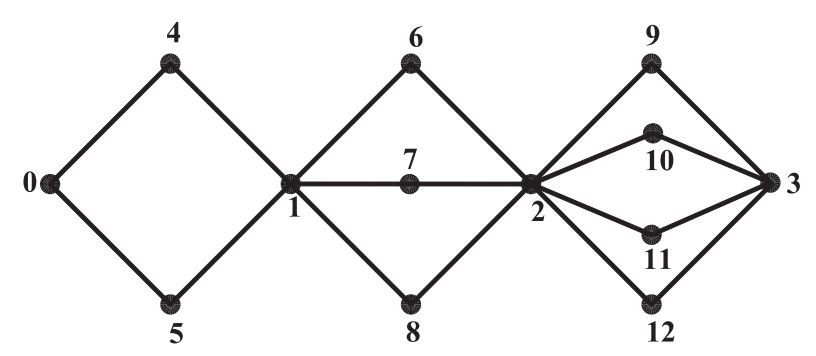

Figure 8. $L^{\prime}(2,1)$-labeling of $P_{4}^{\prime}$

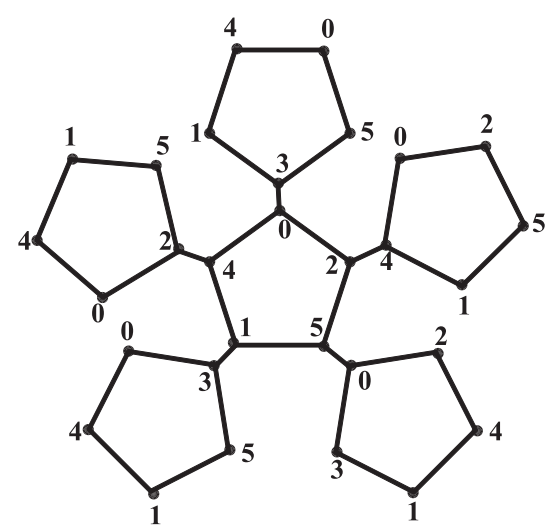

Figure 9. $L(2,1)$-labeling for star of cycle $C_{5}$ 


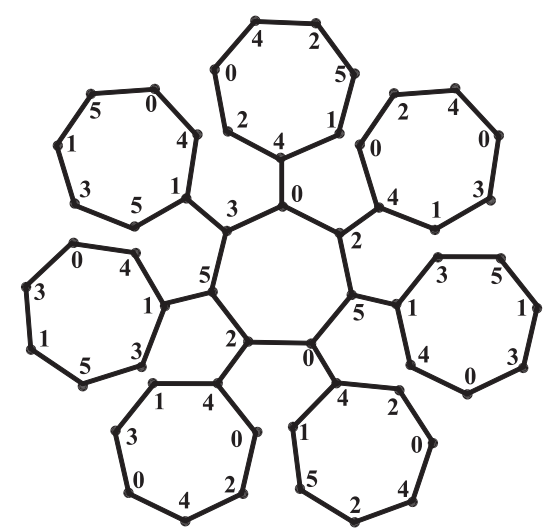

Figure 10. $L(2,1)$-labeling for star of cycle $C_{7}$

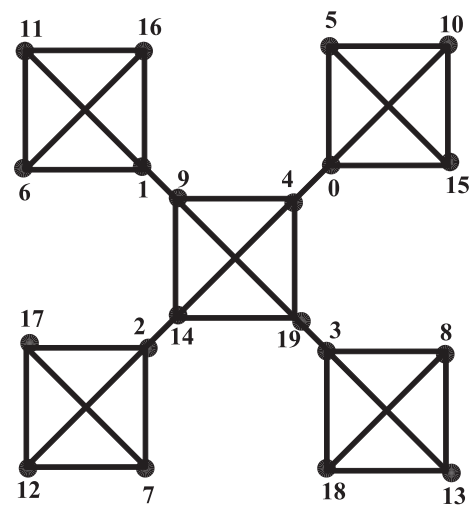

Figure 11. $L^{\prime}(2,1)$-labeling for star of a complete graph $K_{4}$ 